\title{
Vibration behaviour of a bio-composite sandwich with auxetic core
}

\author{
Khawla Essassi ${ }^{1,2, *}$, Jean-Luc Rebiere ${ }^{1}$, Abderrahim El Mahi ${ }^{1}$, Mahamane Toure ${ }^{1}$, Mohamed amine Ben Souf ${ }^{2}$, Anas \\ Bouguecha $^{2}$, and Mohamed Haddar ${ }^{2}$ \\ ${ }^{1}$ Laboratoire d'acoustique de l'université du Mans (LAUM UMR CNRS 6613) Le Mans Université, Av. O. Messiaen 72085 Le Mans \\ Cedex 9, France \\ ${ }^{2}$ Laboratoire de recherche de Mécanique, Modélisation et Production (LA2MP) Ecole Nationale d'Ingénieurs de Sfax, Département \\ Génie Mécanique, Route Soukra, 3038 Sfax, Tunisie
}

\begin{abstract}
This paper describes the flexural vibration and damping performances of an eco-composite sandwich structure with re-entrant honeycomb core. The main objective of this study is to analyse the effect of flax fibre reinforcement composite and the densities of the auxetic core on the dynamic properties of the sandwich structures. The damping properties and the sandwich stiffness are determined in bending beams for different free lengths in a clamped-free configuration. Firstly, the dynamic properties of the skins were investigated in order to develop the evolution of mechanical properties as well as damping coefficient for each material. Then, the same characterization was tested on the sandwich structures with different core densities. The results obtained showed that both core densities and flax fibre as reinforcement plays a major role on the dynamic properties of the sandwich composite structures.
\end{abstract}

\section{Introduction}

In the past decades, eco-composites were widely used in different applications because of the numerous advantages they offer. These materials are ecological, biodegradable and reduce environmental impact. They have also shown an extremely capacity to absorb energy and a high specific strength [1]. Thermoplastic material, such as Polylactic Acid, are also used because of their recyclability [2]. In addition, flax fibre reinforced polymers are the most commonly studied on bio-based composites [3].

To further improve the composite performance, sandwich structures are widely used. Monti et al [4] studied the dynamic behaviour of an eco-sandwich composite structure with Elium/Flax fibre composite as skins and balsa wood as core. In fact, sandwich structures with architectural core were largely studied. The dynamic properties of a sandwich structures with pyramidal truss core have been also studied [5]. The results obtained shown that dynamic properties are improved with the insertion of the viscoelastic layers. More recently, we are witnessing the emergence of auxetic structure because of the unusual properties of becoming thicker when compressed so having negative Poisson's ratios. They showed many advantageous such as having better acoustic properties compared to conventional ones [6] and their ability to dissipate energy [7].

In the present study, sandwich structures with re-entrant honeycomb core are designed and fabricated using 3D printing technology. Free vibration tests are conducted to calculate the natural flexural frequency and the loss factor coefficient. The influence of flax fibre reinforcement and core relative densities on energy absorption of the sandwich composite are also investigated.

\section{Materials and manufacturing}

Sandwich composite were manufactured using two types of bio-based materials, PLA (Polylactic acid) and PFF (PLA flax fibres). These materials are biological and made from renewable materials. Sandwich beams are composed from two skins and re-entrant honeycombs core, as shown in Fig. 1. Sandwich composite is designed using CAD software and then manufactured using 3D printing technology. The length and the width of the printed specimens are respectively $270 \mathrm{~mm}$ and 25 $\mathrm{mm}$. Those with the width of $25 \mathrm{~mm}$ were selected here in order to have different cell numbers in width varying between two and three cells. Two different core densities were tested in this paper. The relative density of the auxetic cell can be calculated as:

$$
\frac{\rho}{\rho_{s}}=\frac{t / l(h / l+2)}{2 \cos \theta(h / l+\sin \theta)}
$$

$\rho$ is the density of the material, $\rho$ s is the density of the auxetic cell, 1 is the original length of the inclined cell walls and $h$ is the length of the vertical ones. $\theta$ is the initial angle between the inclined walls and $\mathrm{X}$ direction.

\footnotetext{
* Corresponding author: khawlaessassi@gmail.com
} 
$\mathrm{b}$ and $\mathrm{t}$ are the specimen thickness and cell wall thickness, respectively. Table 1 present the average dimensions and relative densities of the re-entrant honeycomb cells.

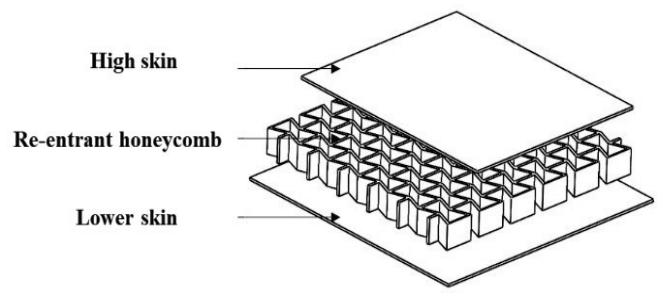

(a)

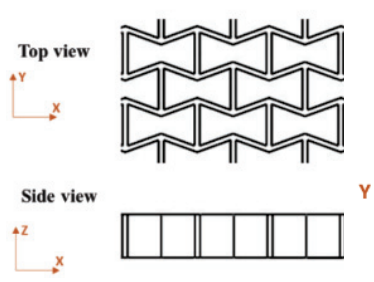

(b)

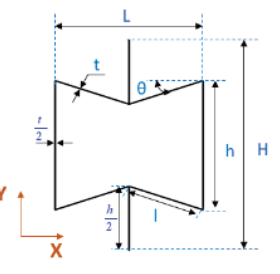

(c)
Fig. 1. a) Sandwich structure with re-entrant honeycomb cores; b) top and side view of the auxetic core and c) design of unit cell of auxetic structure.

Table 1. Design parameters of auxetic core.

\begin{tabular}{|c|c|c|c|c|c|c|}
\hline $\begin{array}{c}\text { Cell } \\
\text { number } \\
\text { in width }\end{array}$ & $\begin{array}{c}\mathrm{l} \\
(\mathrm{mm})\end{array}$ & $\begin{array}{c}\mathrm{h} \\
(\mathrm{mm})\end{array}$ & $\begin{array}{c}\theta \\
(\text { degree })\end{array}$ & $\begin{array}{c}\mathrm{t} \\
(\mathrm{mm})\end{array}$ & $\begin{array}{c}\mathrm{b} \\
(\mathrm{mm})\end{array}$ & $\begin{array}{c}\rho / \rho_{\mathrm{s}} \\
(\%)\end{array}$ \\
\hline 2 cells & 6.65 & 8.52 & -20 & 0.6 & 5 & 16.7 \\
\hline 3 cells & 4.43 & 5.68 & -20 & 0.6 & 5 & 25.1 \\
\hline
\end{tabular}

\section{Experimental setups}

The dynamic characteristics of the sandwich composite structure were evaluated using a free flexural vibration tests, as shown in Fig. 2. Beams were supported horizontally and tested in a clamped-free configuration. The clamping length was set to $40 \mathrm{~mm}$. The free length of the beams was 230,200 , and $170 \mathrm{~mm}$. The specimens were excited near the clamped end using an impact hammer PCB084A14. The responses were detected near the free end using a laser vibrometer. It consists of an OFV 303 sensor head associated with an OFV 3001 controller.

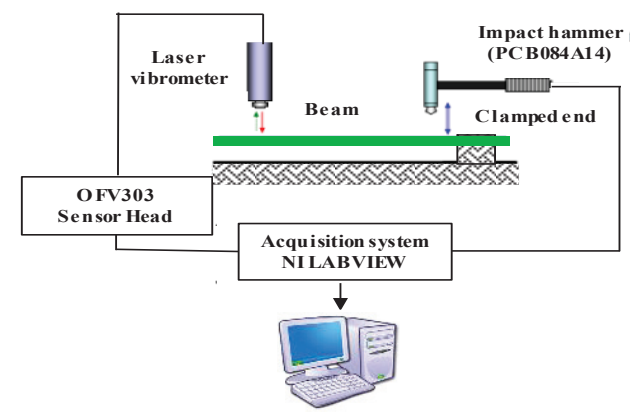

Fig. 2. Experimental equipment for free vibration analysis.
Then, the signals registered from the excitation and from the response were treated by an acquisition card system and analysed with the NVGate software. The dynamic characteristics were calculated using the Fourier transformation of the beam frequency response function to an impulse input. The natural flexural frequencies and the modal loss factor were determined by the Half Power Bandwidth method (HPB), given in Fig. 3, by MATLAB software and used by El Mahi et al. [8]. The resonance frequency fi of the mode $\mathrm{i}$ corresponds to the frequency for which the amplitude of the response is maximum. Using this frequency, the damping loss factor can be calculated by:

$$
\eta_{i}=\frac{\Delta f_{i}}{f_{i}}=\frac{f_{2}-f_{1}}{f_{i}}
$$

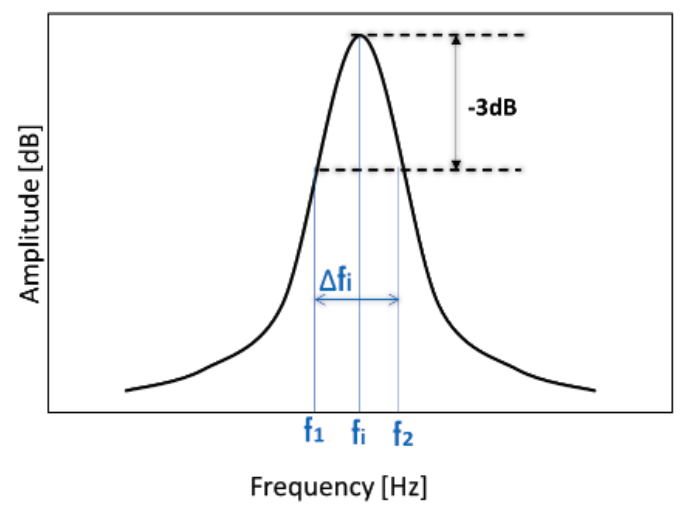

Fig. 3. Half Power Bandwidth method.

The Young modulus E of composite skins and auxetic core for every flexural mode was calculated by:

$$
E=\frac{12 \rho l^{4} f_{n}^{2}}{e^{2} C_{n}^{2}}
$$

With $\rho$ the density of the composite material, 1 the free length of the beam, fn the resonance frequency of the nth flexural mode, e the thickness of the beam in vibration direction and $\mathrm{Cn}$ a coefficient for $\mathrm{nth}$ mode of clampedfree beam, with $\mathrm{C} 1=0.55959, \mathrm{C} 2=3.5069, \mathrm{C} 3=9.8194$ and $\mathrm{Cn}=(\pi / 2)(\mathrm{n}-0.5) 2$ for $\mathrm{n}>3$ [9].

In addition, equivalent stiffness of sandwich beam for every flexural mode can be calculated by:

$$
(E I)_{e q}=\left(\frac{m}{L}\right)\left(2 \pi f_{n}\right)^{2}\left(\frac{l^{2}}{\left(\beta_{n} l\right)^{2}}\right)^{2}
$$

With $\mathrm{m}$ the mass of the beam, $\mathrm{L}$ the total length of the beam, fn the resonance frequency of the nth flexural mode, 1 the free length of the beam and $\beta \mathrm{nl}$ a coefficient for $n$th mode of clamped-free beam: $\beta 11=1.8751, \beta 21=$ $4.6940, \beta 31=7.8547, \beta 41=10.9955, \beta 51=14.1371$ and $\beta n l=(\pi / 2)(2 n-1)$ for $n>5$. 


\section{Results and discussion}

\subsection{Vibration behaviour of composite skins}

Vibration behaviour of PLA and PFF composite skins were determined and given in Fig. 4. It presents the evolution of the loss factor as well as Young Modulus as a function of frequency. Both PFF and PLA exhibit a similar behaviour. The results show that damping loss factor increases when the frequency increases. The loss factor gradually increases in the frequency range from 0 $\mathrm{Hz}$ to $2000 \mathrm{~Hz}$. Above this point, the damping factor still increases slowly to a quasi-asymptotic value. It is clearly seen that the damping coefficients of PLA flax fibre reinforcement are significantly higher than the loss factor of PLA. This difference in loss factor value can be attributed to the viscoelastic nature of elementary flax fibre. As a consequence, the PLA beam is much stiffer than PFF beams. This is clearly shown in the figure given the evolution of the Young Modulus with frequency. For both PLA and PFF specimens, the stiffness determined at low frequency is in a good agreement with the quasi-static Young Modulus.
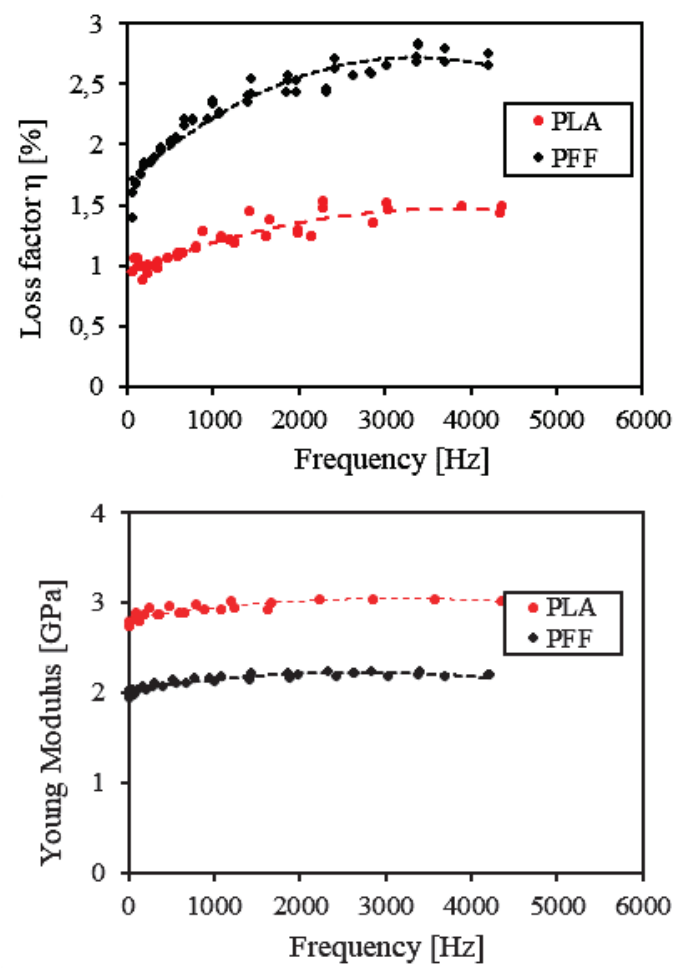

Fig. 4. Evolution of the dynamic properties with frequency of the skins.

\subsection{Vibration behaviour of the sandwich structures}

The vibration behaviour of sandwich beam with auxetic core made PLA and PLA flax fibres were studied for the two core relative densities. The damping factor and the equivalent stiffness were measured with the experimental setup detailed previously.

\subsubsection{Effect of core relative densities}

Fig. 5 present the variation of loss factor and equivalent stiffness as a function of frequency for the PLA sandwich beams. Firstly, it is clearly seen that equivalent stiffness depends on the core densities. It increases with the core relative density and decreases when the frequency increases. It decreases of about $25 \%$ for the two-core relative density in the frequency range from 0 to $4000 \mathrm{~Hz}$. Moreover, the evolution of the damping loss factor with the frequency was given. For each density, the loss factor exhibits an initial increase with frequency. With a frequency range from $0 \mathrm{~Hz}$ to $2000 \mathrm{~Hz}$, there was a close agreement between the two densities results for these loss factor values. The damping coefficient increases about $33 \%$ for the both relative density: $16.7 \%$ and $25.1 \%$. Above this point, the effect of the core densities is clearly observed. It increases with the auxetic core density.
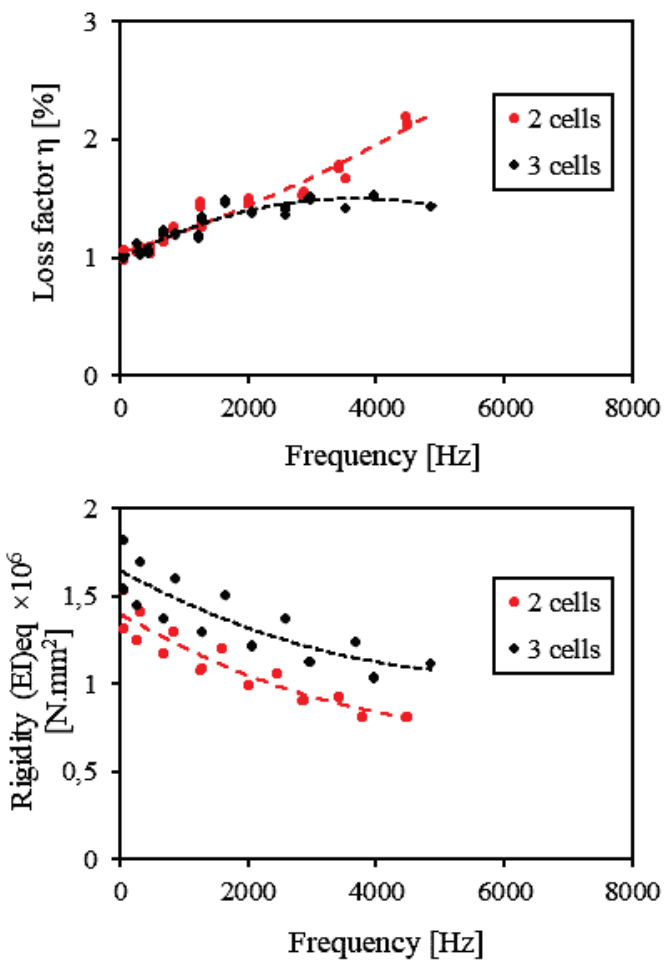

Fig. 5. Evolution of the dynamic properties with frequency of the sandwich structures.

\subsubsection{Flax fibre effect}

The damping behaviour of sandwich beams made of reentrant honeycomb core and PLA and PFF as materials were studied. The flax fibre effect as composite reinforcement were tested for the two relative core density. The equivalent stiffness as well as the damping coefficient of such beams were determined. Fig. 6 show the flax fibre effect on the dynamic properties of the sandwiches. Whatever the core relative density, sandwich beams with flax fibre reinforcement present a highly damping coefficient than those without flax fibre. According to Duc et al. [10], these differences are due to the architectural structure of flax fibre. Indeed, flax fibre structure improve the dissipation of energy by friction of 
the intra-cell wall between cellulose and hemicellulose in each cell wall.

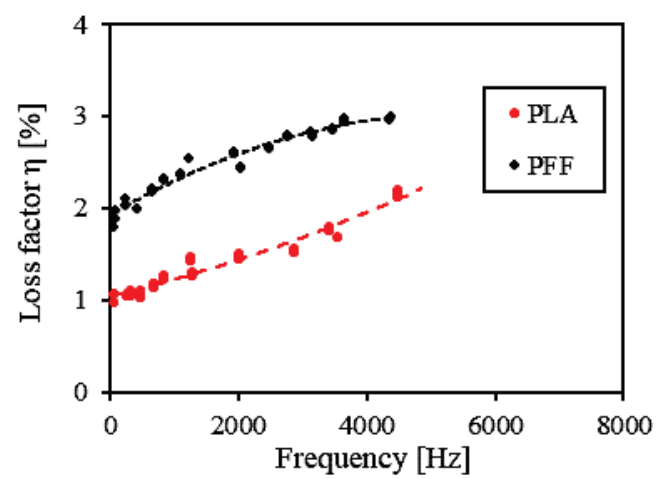

(a)

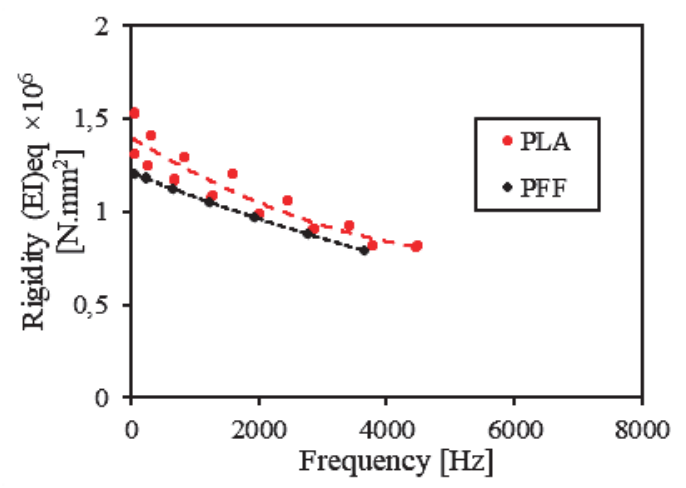

(b)

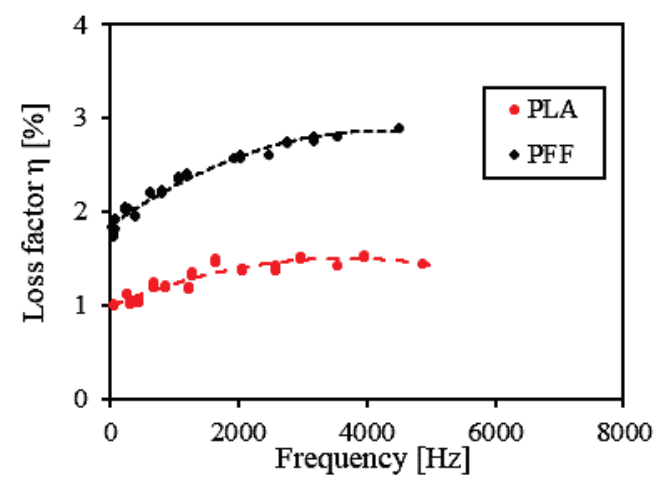

(c)

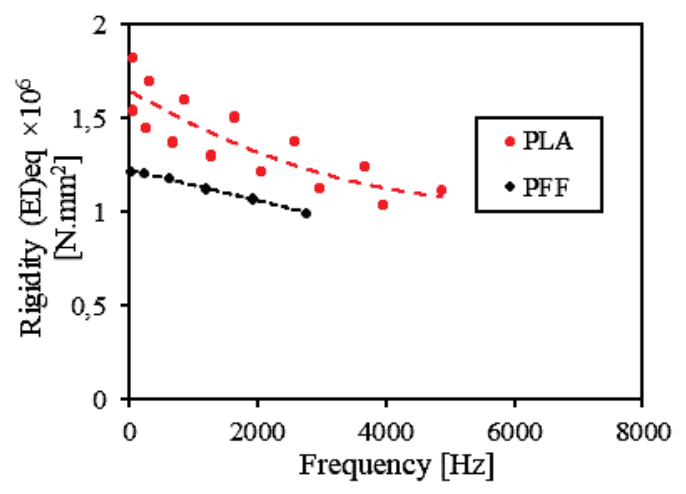

(d)

Fig. 4. Evolution of the dynamic properties with frequency of the sandwich with: (a) and (b) $16.7 \%$ core relative density; (c) and (d) $25.1 \%$ core relative density.

\section{Conclusion}

This study present experimental results of the dynamic properties of a bio-based sandwich structure with auxetic core. The influence of flax fibre as a reinforcement and core densities were investigated. An experimental analysis was affected on each configuration using impulsion technic. Natural flexural frequencies were determined using the Half Power Bandwidth method (HPB)

The damping coefficient deduced from the experimental results present a significant increase as a function of frequency for both PLA and PFF. Besides, flax fibre had a considerable effect on loss factor. It was also shown that beams with flax fibre dissipate more energy than those without reinforcement. In addition, damping coefficient and stiffness properties depend also on the core relative density.

In fact, the obtained results give the dynamic characteristics of the sandwich structure and their constituents. This provide new insights on the development of numerical model which predict the vibration behaviour of such sandwich structures. Such model can be used to further understand the auxetic core form effect. Also, it can be used to optimize the reentrant honeycomb parameters to improve dynamic behaviour as well as increase the energy absorption capacity.

\section{References}

1. L. Yan, B. Kasal, L. Huang, Compos Part B (2016)

2. B. Baghaei, M. Skrifyars, L. Berglin, Compos Part A (2013)

3. J. Zhu, H. Zhu, J. Njuguna, H. Abhyankar, Materials (2013)

4. A.Monti, A. El Mahi, Z. Jendli, L Guillaumat, Compos Part B (2017)

5. J. Yang, J. Xiong, L. Ma, B. Wang, G. Zhang, L. Wu, Compos Struct (2013)

6. C.P. Chen, R.S. Lakes, J. Engineering Materials and Technology (1996)

7. M. Bianchi, F.L. Scarpa, C.W. Smith, J Mater Sci (2008)

8. A. El Mahi, M. Assarar, Y. Sefrani, J.M. Berthelot, Compos Part B (2008)

9. “ASTM E756e98: Standard Test Method for Measuring Vibration Damping Properties of Materials."

10. F. Duc, P.E. Bourban, J.A.E. Manson, Compos Part A (2014) 